\title{
THE HEAT EQUATION ON A COMPACT LIE GROUP
}

\author{
BY
}

H. D. FEGAN ${ }^{1}$

\begin{abstract}
Recently there has been much work related to Macdonald's $\eta$-function identities. In the present paper the aim is to give another proof of these identities using analytical methods. This is done by using the heat equation to obtain Kostant's form of the identities. The basic idea of the proof is to look at subgroups of the Lie group which are isomorphic to the group $S U(2)$. When this has been done the problem has essentially been reduced to that for the group $S U(2)$, which is a classical result.
\end{abstract}

1. Introduction. Let $G$ be a compact, simply connected and connected semisimple Lie group. On $G$ we take the Riemannian structure given by left translation of the metric induced by the Killing form. The Laplacian on $G$ is $\Delta$ and we have chosen the signs so that $\Delta$ has an increasing sequence of positive eigenvalues. We shall take the heat equation in the form

$$
\Delta u-(1 /(2 \pi i)) \partial u / \partial t=0 ;
$$

the coefficient of $\partial u / \partial t$ represents a change of variables so that the variable $t$ lies in the upper half-plane. Now let us define a distribution $\nu(x)$ on $G$ by

$$
\nu(x)=\sum \chi_{\lambda}(x) \chi_{\lambda}(a)
$$

where the summation is over the set of dominant weights, $\chi_{A}$ is the character associated to the dominant weight $\lambda$ and $a$ is an element "principal of type $\rho$ ". We shall use the distribution $\nu(x)$ as initial data for the heat equation (1.1) and denote the solution of this problem by $H_{a}(g, t)$. The main result of this paper is the following product formula for $H_{a}(g, t)$.

THEOREM 1.1. The solution of the heat equation with initial data $\nu(x)$ is

$$
H_{a}(g, t)=\prod_{n=1}^{\infty} \operatorname{det}\left(1-e^{2 \pi i n t} \operatorname{Ad}(g)\right) .
$$

For a definition of an element "principal of type $\rho$ " see $\$ 2$ of this paper or

Received by the editors December 2, 1977.

AMS (MOS) subject classifications (1970). Primary 22C05, 43A75; Secondary 10A20, 35K05.

Key words and phrases. Macdonald's identities, 3 dimensional subgroups of a Lie group, Kostant's element "principal of type $\rho$ ".

'The author was supported by the United Kingdom Science Research Council. 
[11]. It is clear that $a$ is a special point for the heat equation. Let

$$
\phi(t)=e^{-i \pi t / 12} \eta(t)=\prod_{n=1}^{\infty}\left(1-e^{2 \pi i n t}\right)
$$

where $\eta(t)$ is the Dedekind $\eta$-function. Now we shall see that if $H(g, t)$ denotes the fundamental solution of the heat equation then

$$
H(a, t)=H_{a}(1, t)
$$

where 1 is the identity element of $G$. Hence putting $g=1$ gives the result

Proposition 1.2. The fundamental solution of the heat equation satisfies $e^{i \pi k t / 12} H(a, t)=\eta(t)^{k}$ where $k=\operatorname{dim} G$.

The result of this proposition is Kostant's form of Macdonald's identities. In [11] it is obtained from Macdonald's $\eta$-function identities and results about the properties of the element $a$. It can also be proved directly, see [5], and then we have a cycle of three results: Macdonald's $\eta$-function identities, results about the element $a$ and Proposition 1.2, any two of which imply the third. However, Macdonald's identities have a more general form [12, equation (0.4)]. Again there is a cycle of three results: this time the more general form of Macdonald's identities, results about the element $a$ and Theorem 1.1, any two of which imply the third. Thus when we prove Theorem 1.1 directly we shall have given a new proof of Macdonald's identities, this time using the heat equation. Details of the relationship between Theorem 1.1 and Macdonald's identities are given in $\$ 2$ of this paper.

An important example is the case of the group $S U(2)$, where we find a result of Jacobi; see [9]. To explain this we introduce one of the classical theta functions

$$
\theta_{1}(z, t)=2 \sum_{n=0}^{\infty}(-1)^{n} \sin \{(2 n+1) z\} e^{i \pi(n+1 / 2)^{2} t},
$$

see [13]. The result of Jacobi is then

$$
\theta_{1}(z, t)=2 \phi(t) e^{i \pi t / 4} \sin z \prod_{n=1}^{\infty}\left(1-2 e^{2 i \pi n t} \cos 2 z+e^{4 i \pi n t}\right),
$$

see [13]; our notation is consistent with that of [13] where $q$ is used for $e^{i \pi t}$ and $G$ for $\phi(t)$. To see that this result corresponds to Theorem 1.1 we take the expansion of $H_{a}(g, t)$ as a sum of characters which using (1.5) gives

$$
2 e^{i \pi t / 4} \sin (\pi \alpha(g)) H_{a}(g, t)=\theta_{1}(\pi \alpha(g), t)
$$

where $\alpha$ is the unique positive root of $S U(2)$. Substituting this expression into Theorem 1.1 gives the product expansion of $\theta_{1}$. We summarize this in the following result. 
Proposition 1.3. For the group SU(2) the solution of the heat equation with initial data $\nu(x)$ is

$$
H_{a}(g, t)=\theta_{1}(\pi \alpha(g), t) / 2 e^{i \pi t / 4} \sin (\pi \alpha(g)) .
$$

It is convenient to express this in its product form.

Proposition 1.4. For the group SU(2) there is the product formula

$$
H_{a}(g, t)=\phi(t) \prod_{n=1}^{\infty}\left(1-e^{2 \pi i n t} e^{2 \pi i \alpha(g)}\right)\left(1-e^{2 \pi i n t} e^{-2 \pi i \alpha(g)}\right) .
$$

Some details of the case for the group $S U(2)$ will be given in $\$ 3$ of this paper.

Let us now return to considering the more general case of $G$ a compact group. Fix a maximal torus $T$ and choose a set $\{\alpha\}$ of positive roots of $G$ with respect to $T$. Now the adjoint representation has a simple expression in terms of the roots and this enables us to compute explicitly the term $\operatorname{det}\left(1-e^{2 \pi i n t} \operatorname{Ad}(g)\right)$; here 1 is the identity matrix and $\mathrm{Ad}$ is the adjoint representation. Expanding this and then collecting terms by roots gives another expression for our solution of the heat equation.

THEOREM 1.5. The solution of the heat equation with initial data $\nu(x)$ is

$$
H_{a}(g, t)=\phi(t)^{l-\mu} \prod_{\alpha>0}\left\{\frac{\theta_{1}(\pi \alpha(g) ; t)}{2 e^{i \pi t / 4} \sin \pi \alpha(g)}\right\} .
$$

The notation in this theorem is as follows: $l$ is the rank $G, \mu$ is the number of positive roots, the sign $\Pi_{\alpha>0}$ indicates that the product is taken over the positive roots and the other notation is as before.

It is now clear why the example of the group $S U(2)$ is important. Theorem 1.5 expresses our solution of the heat equation as a product of a function of $t$ and then a product of factors each of which is the solution of the heat equation on a copy of the group $S U(2)$. Each of the positive roots of $G$ gives rise to a subgroup isomorphic to $S U(2)$. The heat equation then has a solution which is made up of the product of the solutions on each of these subgroups and a factor $\phi(t)^{l-\mu}$. Since the heat equation is multiplicative, in the sense that if $M=M_{1} \times M_{2}$ is a product manifold with Riemannian structure being the product structure, then $H(x, t)=H_{1}\left(x_{1}, t\right) H_{2}\left(x_{2}, t\right)$ for $x=\left(x_{1}, x_{2}\right)$ and $H, H_{1}$, and $H_{2}$ being the solutions of the heat equation on $M, M_{1}$, and $M_{2}$. Thus one can interpret Theorem 1.5 as expressing the solution of the heat equation on $G$ as a product of the solutions on the subgroups associated to the roots with the extra factor $\phi(t)^{l-\mu}$ to compensate for the fact that $G$ is not a product of these subgroups. In this context Theorem 1.5 gives a partial explanation of Macdonald's identities in terms of the classical identities of Jacobi and the structure of the subgroups isomorphic to $S U(2)$ inside the Lie group. 
We prove Theorem 1.1 in the form of Theorem 1.5. This proof is broken down into a series of stages and is finally completed in $\$ 7$. In $\$ 8$ we give an application of this to give a relation involving the Green's function of the Lie group $G$. The green's function $\gamma$ is the convolution kernel of the operator $\Delta^{-1}$; for details see $\$ 8$ or [7]. The result is

THEOREM 1.6. The Green's function restricted to the torus $T$ satisfies

$$
\nu * \gamma \mid T=\int_{0}^{\infty} \prod_{n=1}^{\infty} \operatorname{det}\left(1-e^{-n t} \operatorname{Ad}(x)\right) d t,
$$

where $\nu * \gamma$ is the convolution of $\gamma$ with the distribution $\nu(x)$.

I wish to thank the many mathematicians with whom I have had helpful conversations, especially Professor S. Helgason who suggested the application of the result given in $\$ 8$ of this paper and Dr. M. Flensted-Jensen for spending much time in discussions.

2. Macdonald's identities. To begin we recall the basic argument for [11]. Macdonald's $\eta$-function identities can be written in the form

$$
\eta(t)^{k}=\sum_{\lambda \in M} d(\lambda+\rho) e^{2 \pi i B(\lambda+\rho)^{2} t}
$$

where $M$ is a suitable lattice, $d(\lambda)=\Pi_{\alpha>0} B(\lambda, \alpha) / \Pi_{\alpha>0} B(\rho, \alpha)$ with the products taken over the positive roots, $\rho=\frac{1}{2} \Pi_{\alpha>0} \alpha$ is half the sum of the positive roots and $B(x, y)$ is the negative of the Killing form. The sign of the Killing form has been changed to make $B(x, y)$ positive definite and we use the notation $B(x)^{2}=B(x, x)$. In the case when $G$ is simply-laced the lattice is $M=h \cdot P$ with $h$ the Coxeter number and $P$ the lattice of weights. It is well known, for example see [10], that $c(\lambda)=B(\lambda+\rho)^{2}-B(\rho)^{2}$ is the eigenvalue of the Casimir operator with corresponding eigenfunctions the matrix coefficients of the representation with highest weight $\lambda$, where we have fixed a fundamental Weyl chamber $D$. Now using the strange formula $B(\rho)^{2}=k / 24$, see [5] or [6], we can rewrite (2.1) as

$$
\phi(t)^{k}=\sum_{\lambda \in M} d(\lambda+\rho) e^{2 \pi i c(\lambda) t}
$$

For $\lambda \in M$ there exists a unique element of $W$ of the Weyl group and a unique element $\mu \in P \cap D$ such that $\lambda=\omega(\mu+\rho)-\rho$; see [11, Lemma 3.5.2]. Hence there is a weighting $\varepsilon(\lambda)$ with $\varepsilon(\lambda)=1,-1$, or 0 such that (2.2) can be written as a sum over the dominant weights. That is

$$
\phi(t)^{k}=\sum_{\lambda \in P \cap D} \varepsilon(\lambda) d(\lambda+\rho) e^{2 \pi i c(\lambda) t} .
$$

Now in [11] the weighting $\varepsilon(\lambda)$ is identified. To do this we must introduce a special element, that is one which is "principal of type $\rho$ ". Let $t$ denote the Lie 
algebra of $T$, the maximal torus which we have chosen in $G$, and $t^{*}$ the dual to $\mathrm{t}$. The Killing form $B(x, y)$ defines an isomorphism $\sigma: \mathrm{t}^{*} \rightarrow \mathrm{t}$. Define an element $a \in T$ by $a=\exp (\sigma(2 \rho))$. For our purposes it is sufficient to define an element "principal of type $\rho$ " up to conjugation; any element conjugate to $a$ will be such an element. The weighting is then found to be $\varepsilon(\lambda)=\chi_{\lambda}(a)$, where $\chi_{\lambda}$ is the character with highest weight $\lambda$. By the Weyl dimension formula $d(\lambda+\rho)=\operatorname{dim} V_{\lambda}=\chi_{\lambda}(1)$ is the dimension of the corresponding representation space. Thus we now have

$$
\phi(t)^{k}=\sum_{\lambda \in P \cap D} \chi_{\lambda}(a) \chi_{\lambda}(1) e^{2 \pi i c(\lambda) t} .
$$

Later we shall discuss solutions of the heat equation. We shall see that this can be represented as a convolution kernel which we denote by $H(x, t)$ and that this has an expression as a sum over characters as

$$
H(x, t)=\sum_{\lambda \in P \cap D} \chi_{\lambda}(x) \chi_{\lambda}(1) e^{2 \pi i c(\lambda) t} .
$$

Thus we have Macdonald's $\eta$-function identities expressed in the form

$$
\phi(t)^{k}=H(a, t)
$$

which is Proposition 1.2. For details of the rearrangement and identification of the weighting $\varepsilon(\lambda)$ used to obtain (2.4) the reader is referred to [11].

Now Macdonald's identities have the more general form, see [12, equation (0.4)], as a formal identity in an indeterminant $X$ :

$$
\prod_{n=1}^{\infty}\left\{\left(1-x^{n}\right)^{l} \prod_{\alpha}\left(1-x^{n} e^{2 \pi i \alpha}\right)\right\}=\sum_{\lambda \in M} \chi_{\lambda} X^{c(\lambda)}
$$

where $\chi_{\lambda}=\Sigma_{\omega \in W}(-1)^{\omega} e^{2 \pi i \omega(\lambda+\rho)} / \Sigma_{\omega \in W}(-1)^{\omega} e^{2 \pi i \omega(\rho)}$. This is of course the Weyl character formula when $\lambda$ is a dominant weight. We now use the action of the Weyl group on the lattice $M$ and as in the case of the special identity we obtain from the right-hand side of (2.7)

$$
\sum_{\lambda \in M} \chi_{\lambda} X^{c(\lambda)}=\sum_{\lambda \in P \cap D} \chi_{\lambda}(a) \chi_{\lambda} X^{c(\lambda)}
$$

Taking $X=e^{2 \pi i t}$ and applying this to $h \in T$ we obtain

$$
\sum_{\lambda \in M} \chi_{\lambda}(h) X^{c(\lambda)}=H_{a}(h, t) .
$$

The steps in the argument to obtain (2.9) are the same as before in the special identity.

To work on the left-hand side of (2.7) we consider the adjoint representation which for $h \in T$ decomposes into:

$$
\operatorname{Ad} h=\left(\begin{array}{lll}
I_{l} & & \\
& A_{\alpha}(h) & \\
& & \ddots
\end{array}\right)
$$


where $I_{l}$ is the $l \times l$ identity matrix and, for each positive root $\alpha, A_{\alpha}(h)$ is the $2 \times 2$ matrix

$$
A_{\alpha}(h)=\left(\begin{array}{rr}
\cos 2 \pi \alpha(\mathbf{h}) & \sin 2 \pi \alpha(\mathbf{h}) \\
-\sin 2 \pi \alpha(\mathbf{h}) & \cos 2 \pi \alpha(\mathbf{h})
\end{array}\right) .
$$

Here $h \in t$ such that $h=\exp h$. This enables us to calculate explicitly

$$
\operatorname{det}\left(1-X^{n} \operatorname{Ad} h\right)=\left(1-X^{n}\right)^{l} \prod_{\alpha>0} \operatorname{det}\left(1-X^{n} A_{\alpha} h\right),
$$

and we see that the right-hand side of (2.7) gives

$$
\prod_{n=1}^{\infty}\left\{\left(1-X^{n}\right)^{l} \prod_{\alpha}\left(1-X^{n} e^{2 \pi i \alpha(\mathrm{h})}\right)\right\}=\prod_{n=1}^{\infty} \operatorname{det}\left(1-X^{n} \operatorname{Ad} h\right) .
$$

Writing $g$ for $h$, putting $X=e^{2 \pi i t}$ and combining (2.7), (2.9) and (2.13) gives

$$
H_{a}(g, t)=\prod_{n=1}^{\infty} \operatorname{det}\left(1-e^{2 \pi i n t} \operatorname{Ad}(g)\right)
$$

which is Theorem 1.1.

3. The group $S U(2)$. The group $S U(2)$ has rank one. The maximal torus is isomorphic to a circle $S^{1}$ and $t \simeq R$. There is one positive root $\alpha$ and we choose an isomorphism $t^{*} \rightarrow \mathbf{R}$ so that $\alpha \rightarrow 1$. With this normalization we have $\rho=\frac{1}{2}$, the Killing form is $B(x, y)=\frac{1}{2} x y$ for $x, y \in \mathbf{R} \cong \mathrm{t}^{*}$. The isomorphism $\sigma: t^{*} \rightarrow t$ induced by the Killing form is a map $\mathbf{R} \rightarrow \mathbf{R}$ and $\sigma(2 \rho)=\frac{1}{2}$. The integer lattice is $2 Z$ and the exponential map is $\exp (x)=\mathrm{e}^{\mathrm{i} \pi \mathrm{x}}$ so $a=e^{i \pi / 2}$. The lattice of weights is $P=\frac{1}{2} Z$ and we take as dominant weights those $\lambda \in \frac{1}{2} \mathbf{Z}$ such that $\lambda>0$. Observe that the Weyl group is $W \cong Z_{2}$ with the nontrivial element acting as multiplication by -1 . By the Weyl character formula the character with highest weight $\lambda$ is

$$
\chi_{\lambda}(g)=\sin ((2 \lambda+1) \pi \mathbf{g}) / \sin (\pi \mathbf{g}),
$$

where $g=\exp (\mathrm{g}), g \in T$ and $\mathrm{g} \in \mathrm{t}$ is regular. The condition $\mathrm{g} \in \mathrm{t}$ is regular is equivalent to $\sin (\pi \mathbf{g}) \neq 0$. It has been noted that $\chi_{\lambda}(a)$ has special importance; the values of this are given as follows.

$$
\chi_{\lambda}(a)= \begin{cases}-1, & \lambda \text { is an odd integer, } \\ 0, & \lambda \text { is not an integer, } \\ 1, & \lambda \text { is an even integer, }\end{cases}
$$

and the eigenvalues of the Casimir operator are

$$
c(\lambda)=\frac{1}{2} \lambda(\lambda+1) \text {. }
$$

The fundamental solution of the heat equation is given by

$$
H(g, t)=\sum_{\lambda \in P \cap D} \chi_{\lambda}(g) \chi_{\lambda}(1) e^{2 \pi i c(\lambda) t},
$$


and the distribution $\nu$ by

$$
\nu(x)=\sum_{n=0}^{\infty}(-1)^{n} \chi_{n}(x) .
$$

Hence the solution $H_{a}(g, t)$, which is obtained by taking the convolution of $\nu(x)$ with $H(g, t)$, is given by

$$
H_{a}(g, t)=\sum_{n=0}^{\infty} \chi_{n}(g)(-1)^{n} e^{2 \pi i c(n) t},
$$

which upon substituting for $\chi_{n}(g)$ becomes

$$
H_{a}(g, t)=\sum_{n=0}^{\infty}(-1)^{n}(\sin \{(2 n+1) \pi \mathrm{g}\} / \sin \pi \mathrm{g}) e^{i \pi n(n+1) t} .
$$

Let us recall the definitions of one of the classical theta functions; from [13] we have

$$
\theta_{1}(z, t)=2 \sum_{n=0}^{\infty}(-1) \sin ((2 n+1) z) e^{i \pi(n+1 / 2)^{2} t} .
$$

Since $n(n+1)=\left(n+\frac{1}{2}\right)^{2}-\frac{1}{4}$ the expression (3.7) can be written as

$$
H_{a}(g, t)=\theta_{1}(\pi \mathrm{g}, t) /\left(2 \sin (\pi \mathrm{g}) e^{i \pi t / 4}\right) .
$$

As before let $\phi(t)=\prod_{n=1}^{\infty}\left(1-e^{2 \pi i n t}\right)$; then there is the classical result, see [13],

$$
\theta_{1}(\pi g, t) /\left(2 \sin (\pi g) e^{i \pi t / 4}\right)=\phi(t) \sum_{n=1}^{\infty}\left(1-2 e^{2 \pi i n t} \cos (2 \pi g)+e^{4 \pi i n t}\right)
$$

Hence by substituting (3.10) into (3.9) we obtain

$$
H_{a}(g, t)=\phi(t) \sum_{n=1}^{\infty}\left(1-e^{2 \pi i n t} e^{2 \pi i g}\right)\left(1-e^{2 \pi i n t} e^{-2 \pi i g}\right) .
$$

This is Proposition 1.4 and equation (3.9) is Proposition 1.3.

Finally observe that since $H(a, t)=H_{a}(1, t)$ by putting $\mathrm{g}=0$ we obtain the special identity for $S U(2)$, that is

$$
H(a, t)=\phi(t)^{3} .
$$

4. The heat equation. We begin this section with some results about the heat equation on a Riemannian manifold. After discussing it in this generality we shall then specialize to the case when the Riemannian manifold is a Lie group. Since much of this is taken from [1] and [2] the reader is referred there for proofs and further details.

Let $M$ be a compact Riemannian manifold with metric tensor $g_{i j}$; then in 
local coordinates the Laplacian is given by

$$
\Delta f=\frac{-1}{\sqrt{\operatorname{det}\left(g_{i j}\right)}} \sum_{r, s} \frac{\partial}{\partial x^{r}}\left(\sqrt{\operatorname{det}\left(g_{i j}\right)} a^{r s} \frac{\partial f}{\partial x^{s}}\right),
$$

where $\left(g^{r s}\right)=\left(g_{i j}\right)^{-1}$. As the heat equation on $M$ we take $\Delta G+\partial G / \partial t=0$, $G(x, 0)=f(x)$, where $G: M \times \mathbf{R}_{+} \rightarrow \mathbf{R}$ is the solution to the problem with $f$ : $M \rightarrow \mathbf{R}$ a given initial condition. This problem can be solved for any initial condition $f$ when the kernel of the heat equation is known.

Definition. The kernel of the heat equation is a function

$$
K: M \times M \times \mathbf{R}_{+} \rightarrow \mathbf{R}
$$

satisfying the following three conditions:

(1) $K$ is $C^{0}$ in the three variables, $C^{2}$ in the first two variables and $C^{1}$ in the last variable.

(2) $\Delta_{2} K+\partial K / \partial t=0$ where $\Delta_{2}$ is the Laplacian acting on the second variable.

(3) For each $y \in M, \lim _{t \rightarrow 0} K(x, y, t)=\delta_{y}(x)$, where $\delta_{y}(x)$ is the Dirac measure concentrated at $y$.

We need the following normalization of measures on $M$. The metric tensor $g_{i j}$ defines a volume form and hence a measure $d g$ on $M$. Let $v=\int_{M} 1 d g$ so that $v$ is the volume of $M$ with respect to the Riemannian structure and set $d x=v^{-1} d g$ so that $d x$ is a measure on $M$ with $\int_{M} 1 d x=1$. The Dirac measure concentrated at $y$ then has the formal property that, for a suitable class of functions $f: M \rightarrow \mathbf{R}, \int_{M} f(x) \delta_{y}(x) d x=f(y)$.

It is known that the kernel of the heat equation exists and is unique. Once we have such a kernel then the solution of the heat equation with initial condition $f$ is given by

$$
G(y, t)=\int_{M} f(x) K(x, y, t) d x .
$$

The function $K$ has an expansion in terms of the eigenfunctions of the Laplacian. Let $\operatorname{spec}(M)$ denote the eigenvalues of the Laplacian $\Delta$ and for $\lambda \in \operatorname{spec}(M)$ let $\psi_{1}, \ldots, \psi_{r}$ be an orthonormal basis of the eigenfunctions of $\Delta$ with eigenvalue $\lambda$ and using $\langle\psi, \phi\rangle=\int_{M} \psi(x) \phi(x) d x$ as the inner product. Change notation so that $\left\{\phi_{j}\right\}$ is a complete set of orthonormal eigenfunctions of $\Delta$ and let $\lambda_{j}$ be the eigenvalue corresponding to $\phi_{j}$. Of course these are not all distinct but we have an increasing sequence

$$
0 \leqslant \lambda_{1} \leqslant \lambda_{2} \leqslant \ldots
$$

which is unbounded. With this notation $K$ is given by

$$
K(x, y, t)=\sum \phi_{j}(x) \phi_{j}(y) e^{-\lambda_{j} t} .
$$

It is clear from this formula that $K(x, y, t)=K(y, x, t)$ and that $K$ is $C^{\infty}$ in 
the three variables and analytic in the third.

Now let $M=G$ be a compact simply connected Lie group. Let $g_{i j}$ be the bi-invariant metric defined by the innerproduct induced from the Killing form. The Casimir operator is denoted by $\Delta$ and we adopt the convention of sign which gives $\Delta$ an increasing sequence of positive eigenvalues. We shall call the function given by the following definition the fundamental solution.

DEFINITION. The fundamental solution of the heat equation is

$$
H: G \times \mathbf{R}_{+} \rightarrow \mathbf{R}
$$

which satisfies the three conditions:

(1) $H$ is $C^{0}$ in the two variables, $C^{2}$ in the first and $C^{1}$ in the second.

(2) $\Delta H+\partial H / \partial t=0$.

(3) $\lim _{t \rightarrow 0} H(x, t)=\delta_{1}(x)$ where $\delta_{1}(x)$ is the Dirac measure concentrated at the identity of the group $G$.

Again we use a normalized measure. Let $d x$ denote the unique bi-invariant Haar measure on $G$ normalized so that $\int_{G} l d x=1$. Then if $f: G \rightarrow \mathbf{R}$ is an initial condition the corresponding solution of the heat equation is given by

$$
G(y, t)=\int_{G} f(x) H\left(x^{-1} y, t\right) d x .
$$

A proof of this follows immediately from the next theorem.

THEOREM 4.1. The functions $H$ and $K$ are related by $H\left(x^{-1} y, t\right)=$ $K(x, y, t)$. In particular since $K$ exists and is unique so is $H$.

Proof. Since $K(x, y, t)$ satisfies the conditions of the first definition and is unique we can define $H(y, t)=K(1, y, t)$, and then check that $H$ satisfies the conditions of the second definition. Conversely for each $H$ satisfying the second definition let $K(x, y, t)=H\left(x^{-1} y, t\right)$ and we see that $K$ satisfies the first definition and hence $H$ is unique.

The function $K$ has an expansion as a sum of eigenfunctions of $\Delta$; see (4.4). There is an analogous expansion for $H$ in terms of the characters of $G$. For $\lambda \in P \cap D$ a dominant weight let $\pi_{\lambda}: G \rightarrow$ Aut $V_{\lambda}$ denote the corresponding finite dimensional irreducible representation and $\chi_{\lambda}$ the character of $\pi_{\lambda}$. It is well known that the Casimir element $\Delta \in Z(\mathfrak{U})$ is an element of the centre of the universal enveloping algebra $\mathfrak{U}$ of $\mathfrak{g}$. Hence $\Delta$ acts by scalar multiplication on the representation and this scalar is $c(\lambda)$; see [10]. Thus the eigenfunctions of $\Delta$ are the matrix coefficients of $\pi_{\lambda}$ and the corresponding eigenvalue is $c(\lambda)$. This gives the following expansion in characters for $H$ :

$$
H(g, t)=\sum_{\lambda \in P \cap D} \chi_{\lambda}(1) \chi_{\lambda}(g) e^{-c(\lambda) t} .
$$

Notice that this follows from the Peter-Weyl theory which gives the following. 
Let

$$
S=\underset{\lambda \in P \cap D}{\bigoplus}\left(V_{\lambda} \otimes V_{\lambda}^{*}\right) ;
$$

then $S$ is dense in $L^{2}(G)$, the square-integrable functions on $G$. In this $V_{\lambda}^{*}$ is the dual space to $V_{\lambda}$.

We have chosen a normalization of our characters so that $\chi_{\lambda}(1)=\operatorname{dim} V_{\lambda}$. This gives us the following formula for the convolution of characters:

$$
\chi_{\lambda}(1)\left(\chi_{\lambda} * \chi_{\mu}\right)(g)= \begin{cases}0 & \text { if } \lambda \neq \mu, \\ \chi_{\lambda}(g) & \text { if } \lambda=\mu .\end{cases}
$$

For the case which we are studying we wish to work with the solution of the heat equation with initial data $\nu(x)$. Recall that $\nu(x)$ was defined as a sum of characters

$$
\nu(x)=\sum \chi_{\lambda}(a) \chi_{\lambda}(x)
$$

where $a$ is an element "principal of type "principal of type $\rho$ " and so $\chi_{\lambda}(a)$ is $+1,-1$ or 0 . The solution with initial data $\nu(x)$ is given by

$$
H_{a}(g, t)=(\nu * H)(g, t)
$$

and so using (4.8) and the expressions for $\nu$ and $H$ as sums of characters we obtain immediately

$$
H_{a}(g, t)=\sum_{\lambda \in P \cap D} \chi_{\lambda}(a) \chi_{\lambda}(g) e^{-c(\lambda) t} .
$$

Notice that from equations (4.6) and (4.11) we have

$$
H(a, t)=H_{a}(1, t) \text {. }
$$

Finally we remark that in our applications we shall replace the variable $t$ by $-2 \pi i t$. This corresponds to replacing the heat equation by the equation $\Delta u+(-1 / 2 \pi i) \partial u / \partial t=0$ when the variable $t$ lies along the imaginary axis in the upper half-plane. Then we shall replace the solutions by their analytic continuations to the whole upper half-plane. Neither of these steps presents any difficulties and making this change helps us to relate our functions to the classical ones which are traditionally defined on the upper half-plane.

5. The heat equation on a torus. To study the fundamental solution on the maximal torus $T$ we define an embedding into another torus $T^{\mu}$. Consider a positive root $\alpha$; then $\alpha: t \rightarrow \mathbf{R}$ is a linear functional on the Lie algebra of $T$. Since $\alpha$ takes integer values on the integer lattice there is associated to $\alpha$ a map $\alpha_{*}: T \rightarrow \mathbf{R} / \mathbf{Z}$. Let $\left\{\alpha_{1}, \ldots, \alpha_{\mu}\right\}$ be the set of positive roots, where $\mu$ is the number of positive roots, and let $T^{\mu}=S^{1} \times \cdots \times S^{1}$ with $\mu$ factors. Then the embedding $A_{*}: T \rightarrow T^{\mu}$ is given by

$$
A_{*}(x)=\left(\alpha_{1^{*}}(x), \ldots, \alpha_{\mu^{*}}(x)\right) \text {. }
$$


Similarly we have a map $A: t \rightarrow \mathbf{R}^{\mu}$ of Lie algebras which is given by

$$
A(\mathbf{x})=\left(\alpha_{1}(\mathbf{x}), \ldots, \alpha_{\mu}(\mathbf{x})\right)
$$

for $x \in t$ and where we are now regarding the roots as linear functionals on $t$. In fact since the map $\alpha_{j}$ is the derivative, at the identity element, of $\alpha_{j^{*}}$ then the map $A$ is the derivative of $A_{*}$.

We shall show that in a natural way the $\operatorname{map} A_{*}$ is an isometry. To do this we must introduce Riemannian metrics on $T$ and $T^{\mu}$. Firstly introduce inner products on $t$ and $R^{\mu}$ as follows. For $x, y \in t$ let $B(x, y)=\Sigma \alpha(x) \alpha(y)$ be the negative of the Killing form, with the sum over all the roots both positive and negative. Since $G$ is a compact semisimple Lie group $B$ is a nondegenerate positive definite innerproduct on $\mathrm{t}$. For $u, v \in \mathbf{R}^{\mu}$ with $u=\left(u_{1}, \ldots, u_{\mu}\right)$ and $v=\left(v_{1}, \ldots, v_{\mu}\right)$ let $\langle u, v\rangle=2 \Sigma u_{j} v_{j}$ be twice the standard innerproduct on $\mathbf{R}^{\mu}$. Now $t$ and $\mathbf{R}^{\mu}$ are the tangent spaces at the identity to $T$ and $T^{\mu}$. Hence by left translation these innerproducts define Riemannian structures on $T$ and $T^{\mu}$. With these Riemannian structures the map $A_{*}$ is an isometry.

LEMMA 5.1. The embedding $A_{*}: T \rightarrow T^{\mu}$ is an isometry.

Proof. It is sufficient to check that $B(\mathbf{x}, \mathbf{y})=\langle A(\mathbf{x}), A(\mathbf{y})\rangle$ since $A$ is the derivative of $A$. This follows immediately from the definitions since $B(\mathbf{x}, \mathbf{y})=$ $2 \sum_{j=1}^{\mu} \alpha_{j}(\mathbf{x}) \alpha_{j}(\mathbf{y})$.

The main result of this section can now be stated and proved.

THEOREM 5.2. Let $h(x, t)$ and $h_{\mu}(x, t)$ denote respectively the fundamental solutions of the heat equation on $T$ and $T^{\mu}$; then there is a function $F_{1}(t)$ such that $h(x, t)=F_{1}(t) h_{\mu}\left(A_{*}(x), t\right)$.

Proof. Since the embedding $A_{*}$ is an isometry which preserves the group structure, that is $A_{*}(x y)=A_{*}(x) A_{*}(y)$, it is sufficient to check the result infinitesimally. Thus if $\mathbf{h}(\mathbf{x}, t)$ and $\mathbf{h}_{\mu}(\mathbf{x}, t)$ denote the liftings of $h(x, t)$ and $h_{\mu}(x, t)$ it is sufficient to check that

$$
\mathbf{h}(\mathbf{x}, t)=F_{1}(t) \mathbf{h}_{\mu}(\mathbf{x}, t) .
$$

To do this let $\mathbf{y}_{1}, \ldots, \mathbf{y}_{l}$ be an orthonormal basis for $\mathrm{t}$ with respect to $B(\mathbf{x}, \mathbf{y})$; then $\mathrm{z}_{1}=A\left(\mathbf{y}_{1}\right), \ldots, \mathbf{z}_{l}=A\left(\mathbf{y}_{l}\right)$ are orthonormal in $\mathbf{R}^{\mu}$ with respect to $\langle\mathbf{x}, \mathbf{y}\rangle$. Complete these to an orthonormal basis $\mathbf{z}_{1}, \ldots, \mathbf{z}_{\mu}$ for $\mathbf{R}^{\mu}$. Then since the heat equation is multiplicative

$$
\mathbf{h}(\mathbf{x}, t)=\prod_{j=1}^{l} \mathbf{h}_{1}\left(y_{j}(\mathbf{x}), t\right)
$$

and

$$
\mathbf{h}_{\mu}(\mathbf{x}, t)=\prod_{j=1}^{\mu} \mathbf{h}_{1}\left(z_{j}(\mathbf{x}), t\right)
$$


where the functions $y_{j}(\mathbf{x})$ are given by $\mathbf{x}=\Sigma y_{j}(\mathbf{x}) \mathbf{y}_{j}$ and similarly for $z_{j}(\mathbf{x})$. In (5.4) and (5.5) $\mathbf{h}_{1}(x, t)$ denotes the lifting of the solution on a circle. From the definition of these bases we have

$$
z_{j}(A(\mathbf{x}))= \begin{cases}y_{j}(\mathbf{x}) & \text { for } j=1, \ldots, l, \\ 0 & \text { for } j=l+1, \ldots, \mu .\end{cases}
$$

Thus the result of Theorem 5.2 follows with $F_{1}(t)=\mathbf{h}_{1}(0, t)^{l-\mu}$.

REMARK. We have in fact proved more than is stated in the theorem by identifying $F_{1}(t)$ as $F_{1}(t)=\mathbf{h}_{1}(0, t)^{l-\mu}$.

6. Passage between the torus and the group. Let $\Delta_{G}$ denote the Laplacian on $G$ and $\Delta_{T}$ the flat Laplacian on $T$, both of which are associated to the metrics induced by the Killing form. As before we have chosen the signs so that both $\Delta_{G}$ and $\Delta_{T}$ have increasing sequences of positive eigenvalues. If $f$ is a class function on $G$, that is $y=g x g^{-1}$ implies $f(y)=f(x)$, then $f$ is determined by its restriction $f \mid T$ to the maximal torus. Similarly $\Delta_{G}(f)$ is also a class function and so is determined by $\left(\Delta_{G} f\right) \mid T$. The two functions $\left(\Delta_{G} f\right) \mid T$ and $\Delta_{T}(f \mid T)$ are in fact related and the result is due to Harish-Chandra.

LEMMA 6.1. The functions $\left(\Delta_{G} f\right) \mid T$ and $\Delta_{T}(f \mid T)$ are related by

$$
j(x)\left(\Delta_{G} f\right)(x)=\left(\Delta_{T}-B(\rho)^{2}\right) j(x)(f \mid T)(x),
$$

where $j(x)=\Pi_{\alpha>0}\left(2 i \sin \pi \alpha_{*}(x)\right)$ is the denominator of the Weyl character formula and as usual $B(\rho)^{2}$ means $B(\rho)^{2}=B(\rho, \rho)$.

Proof. See [7, p. 587], but notice that the signs of our Laplacians are different to those in [7], hence the difference in the sign of $B(\rho)^{2}$.

As a consequence of this we can express solutions of the heat equation on $G$, restricted to $T$, in terms of solutions of the flat heat equation on $T$. More precisely this is given by the result.

Proposition 6.2. Let $k_{T}(x, t)$ be a solution of the heat equation on $T$; then for $x$ a regular element of $T$

$$
k_{G}(x, t)=e^{-2 \pi i B(\rho)^{2} t} k_{T}(x, t) / j(x)
$$

is a solution of the heat equation on $G$ restricted to the regular elements of $T$.

Proof. This follows from an easy calculation by substituting the functions into the equation $\Delta u-(1 / 2 \pi i) \partial u / \partial t=0$, with the relevant Laplacian $\Delta$.

It is important to realize that this result gives a correspondence between solutions of the heat equation on $T$ with those on $G$ rather than between fundamental solutions. In fact if $k_{T}(x, t)$ is the fundamental solution on $T$ then the corresponding function $k_{G}(x, t)$ is not the fundamental solution of the equation on $G$. This is clearly seen by observing that on a manifold $M$ if $H(x, t)$ denotes the fundamental solution then $\lim _{t \rightarrow i \infty} H(x, t)=1$. Hence if 
$k_{T}(x, t)$ is the fundamental solution we have that $k_{G}(x, t)$ satisfies as $t \rightarrow i \infty$ the following:

$$
\lim _{t \rightarrow i \infty} k_{G}(x, t)=\lim _{t \rightarrow i \infty} e^{-2 \pi i B(\rho)^{2} t} / j(x)
$$

and so $k_{G}(x, t)$ is unbounded.

We are interested in finding the solution of the heat equation on $T$ which corresponds to the solution on $G$ with initial data $\nu(x)$. For convenience of notation we shall call this the $\nu(x)$-solution; more precisely we make the following definition.

DEFINITION. If $H_{a}(g, t)$ is the solution of the heat equation on $G$ with initial data $\nu(x)$ then the function

$$
k(x, t)=e^{2 \pi i B(\rho)^{2} t} j(x) H_{a}(x, t)
$$

for $x$ a regular element of the maximal torus $T$ will be called the $\nu(x)$-solution of the heat equation on $T$.

One way to describe the $\nu(x)$-solution is to consider its expansion in a series of characters. It has already been shown that

$$
H_{a}(x, t)=\sum_{\lambda \in P \cap D} \chi_{\lambda}(x) \chi_{\lambda}(a) e^{2 \pi i c(\lambda) t} .
$$

Hence since $c(\lambda)=B(\lambda+\rho)^{2}-B(\rho)^{2}$ we have from the definition

$$
K(x, t)=\sum_{\lambda \in P \cap D} j(x) \chi_{\lambda}(x) \chi_{\lambda}(a) e^{2 \pi i B(\lambda+\rho)^{2} t} .
$$

Now the Weyl character formula gives for $x$ a regular element of $T$ with $x=\exp \mathbf{x}, \mathbf{x} \in \mathrm{t}$

$$
j(x) \chi_{\lambda}(x)=\sum_{\omega \in W}(-1)^{\omega} e^{2 \pi i \omega(\lambda+\rho)(\mathbf{x})} .
$$

Before substituting this into equation (6.4) observe that under the action of the Weyl group the orbit of $P \cap D$ in $P$ is in fact the whole of $P$. We must now consider the function $\chi_{\lambda}(a)$ as a function of $\lambda$. By the Weyl character formula we have

$$
j(a) \chi_{\lambda}(a)=\sum_{\omega \in W}(-1)^{\omega} e^{2 \pi i B(\omega(\lambda+\rho), 2 \rho)}
$$

where we have used the definition of $a=\exp (\sigma(2 \rho))$; see $\$ 2$. Now since the Killing form $B(x, y)$ is invariant under the action of the Weyl group we can write (6.6) as

$$
j(a) \chi_{\lambda}(a)=\sum_{\omega \in W}(-1)^{\omega} e^{2 \pi i B(\omega \rho, 2(\lambda+\rho))} .
$$

At this point we can use the Weyl denominator formula to observe that we 
have

$$
j(a) \chi_{\lambda}(a)=j(2 \sigma(\lambda+\rho)),
$$

where, as in $\$ 2, \sigma: t^{*} \rightarrow \mathrm{t}$ is induced by the Killing form and the function $j$ has, on the right-hand side of (6.8), been lifted from $T$ to $t$. In [11] it is shown that $a$ is a regular element of $T$ and so $j(a) \neq 0$. Define the function a: $\mathfrak{t}^{*} \rightarrow \mathbf{R}$ by

$$
\mathrm{a}(\lambda)=j(2 \sigma(\lambda)) / j(a)
$$

so that $\chi_{\lambda}(a)=\mathbf{a}(\lambda+\rho)$. This function has the properties:

(1) $\mathrm{a}(\lambda)=0$ if $\lambda$ is in a wall of the Weyl chamber,

(2) $\mathrm{a}(\omega \lambda)=(-1)^{\omega} a(\lambda)$ for $\omega \in W$.

Here $(-1)^{\omega}$ is the sign of $\omega$. Since $\rho \in P$ there is a map $P \rightarrow P$ given by $\lambda \rightarrow \lambda+\rho$ and it is well known that under this map $P \cap D$ maps onto the lattice points in the interior of $D$. Using these facts we can substitute the Weyl character formula, (6.5), into equation (6.4) to obtain the following result.

LEMMA 6.3. If $x \in T$ is a regular point and $\mathrm{x} \in \mathrm{t}$ satisfies $x=\exp \mathrm{x}$ then

$$
K(x, t)=\sum_{\lambda \in P} a(\lambda) e^{2 \pi i\left(\lambda(x)+B(\lambda)^{2} t\right)} .
$$

COROLlaRY 6.4. Let $\nu^{1}$ be the distribution on $T$ defined by $\nu^{1}(x)=$ $\sum_{\lambda \in P} a(\lambda) e^{2 \pi i \lambda(x)}$; then $K(x, t)$ is the solution of the heat equation on $T$ with initial condition $\nu^{1}(x)$.

In the previous section the fundamental solution of the heat equation on the torus $T$ was expressed as a product over the roots, this is essentially the content of Theorem 5.2. We shall express the solution on $G$ with initial data $\nu(x)$ as a product over the roots.

THEOREM 6.5. Let $H_{a}(x, t)$ denote our solution of the heat equation on $G$; then there is a function $F(t)$ such that

$$
H_{a}(x, t)=F(t) \prod_{\alpha>0} H_{3}\left(\alpha_{*}(x), t\right)
$$

for $x \in T$ where $H_{3}(x, t)$ is the solution of the heat equation on $S U(2)$.

Proof. Our solution $H(x, t)$ is related to the $\nu(x)$-solution $K(x, t)$ by the definition

$$
K(x, t)=e^{2 \pi i B(\rho)^{2} t} j(x) H(x, t) .
$$

Now by the "strange formula", see [6], $B(\rho)^{2}=k / 24$ where $k=\operatorname{dim} G$, but $k=l+2 \mu$ and so

$$
B(\rho)^{2}=(l-\mu) / 24+3 \mu / 24
$$


By Weyl's denominator formula we can express $j(x)$ in terms of $j_{3}(x)$, the denominator of the Weyl character formula for $S U(2)$, this is

$$
j(x)=\prod_{\alpha>0} j_{3}\left(\alpha_{*}(x)\right) \text {. }
$$

Since $S U(2)$ is three dimensional and we have $\mu$ positive roots we can write (6.10) as

$$
K(x, t)=e^{2 \pi i(l-\mu) t / 24} H(x, t) \prod_{\alpha>0} e^{2 \pi i 3 t / 24} j_{3}\left(\alpha_{*}(x)\right),
$$

and so it is sufficient to prove that

$$
K(x, t)=e^{2 \pi i(l-\mu) t / 24} F(t) \prod_{\alpha>0} K_{3}\left(\alpha_{*}(x), t\right)
$$

where $K_{3}$ is the $\nu(x)$-solution of the group $S U(2)$.

The solution of the heat equation on $T$ can be obtained from the initial condition and the fundamental solution. To complete the proof of the theorem we must study both the initial condition given by $\nu^{1}(x)$ and the fundamental solution.

To consider the distribution $\nu^{1}(x)$ let us recall its definition:

$$
\nu^{1}(x)=\sum_{\lambda \in P} a(\lambda) e^{2 \pi i \lambda(x)}
$$

where $a(\lambda)=j(\sigma(2 \lambda)) / j(a)$. Now the Weyl denominator function is the product over the roots

$$
j(x)=\prod_{\alpha>0} j_{3}(\alpha(x))
$$

of the corresponding function for the group $S U(2)$. Thus we have that $a(\lambda)$ is the product over the roots

$$
a(\lambda)=\prod_{\alpha>0} a_{3}(B(\alpha, \lambda))
$$

again this is a product of the corresponding function for the group $S U(2)$. Now $a(\lambda)$ is, by (6.15), the Fourier transform of $\nu^{1}(x)$. Thus the product formula (6.17) for $a(\lambda)$ gives an expression for $\nu^{1}(x)$ as the convolution product of the corresponding functions for $S U(2)$ with the product taken over the positive roots. Now from Theorem 5.2 we have

$$
h(x, t)=F_{1}(t) \prod_{\alpha>0} h_{1}\left(\alpha_{*}(x), t\right),
$$

where $h(x, t)$ is the fundamental solution on $T$ and $h_{1}$ is the fundamental solution on a circle. The product formula (6.17) now enables us to use the 
argument of Theorem 5.2 and so obtain the product formula for the $\nu(x)$ solution. This is

$$
K(x, t)=F_{2}(t) \prod_{\alpha>0} K_{3}(\alpha(x), t)
$$

for $F_{2}(t)$ a suitable function of $t$.

The simply change in notation

$$
F_{2}(t)=e^{2 \pi i(l-\mu) t / 24} F(t)
$$

gives equation (6.14) which completes the proof of the theorem.

REMARK. As in Theorem 5.2 we have in fact proved more than is stated in the theorem. The additional information is that $F(t)=f(t)^{l-\mu}$ for some function $f(t)$. In Theorem 5.2 we were able to express the corresponding function in terms of the fundamental solution of the heat equation on a circle. Here we shall only remark that $f(t)$ is related to a solution on the circle and determine it in detail later.

7. Proof of the main theorem. In this section we shall complete the proof of the main theorem, that is Theorem 1.1, which we shall obtain in the form of Theorem 1.5. That is we shall prove the following

THEOREM 7.1. The solution of the heat equation on the Lie group $G$ with initial data $\nu(x)$ is

$$
H_{a}(x, t)=\phi(t)^{l-\mu} \prod_{\alpha>0} \frac{\theta_{1}(\pi \alpha(\mathbf{x}), t)}{2 \sin (\pi \alpha(\mathbf{x})) e^{i \pi t / 4}} .
$$

Proof. Recall the equations we have already established.

$$
\begin{gathered}
H_{a}(x, t)=F(t) \prod_{\alpha>0} H_{3}\left(\alpha_{*}(x), t\right), \\
H_{3}(x, t)=\frac{\theta_{1}\left(\pi \alpha_{*}(x), t\right)}{2 \sin \pi \alpha_{*}(x) e^{i \pi t / 4}}
\end{gathered}
$$

and the function $F(t)$ has the form

$$
F(t)=f(t)^{l-\mu} \text {. }
$$

These are from Theorem 6.5, equation (3.9) and equation (6.20).

In [5] it was shown that

$$
H(a, t)=\phi(t)^{k}
$$

Hence since $H(a, t)=H_{a}(1, t)$ we see that

$$
f(t)^{l-\mu} \phi(t)^{3 \mu}=\phi(t)^{l+2 \mu}
$$

where we have used the identity $k=l+2 \mu$. It is immediate from (7.5) that $f(t)=\phi(t)$ which completes the proof of the theorem. 
Alternatively to see that $f(t)=\phi(t)$ we can recall equation (3.12) which gives

$$
H_{3}(1, t)=\phi(t)^{3} \text {. }
$$

Now from the proof of Theorem 6.5 we see that $f(t)$ comes from the solution evaluated at the origin. That is we see that

$$
H_{a}(1, t)=f(t)^{k} \text {. }
$$

Comparing this with the result for $S U(2)$, equation (7.6), gives that for some constant $c$, a root of unity,

$$
f(t)=c \phi(t)
$$

To determine $c$ we let $t \rightarrow i \infty$ and we find $c^{l-\mu}=1$ and so it is sufficient to take $f(t)=\phi(t)$.

8. The Green's function on $G$. Consider the following space of functions on G,

$$
C_{0}^{\infty}(G)=\left\{f: \int f(x) d x=0\right\} .
$$

Then the Laplacian $\Delta: C_{0}^{\infty}(G) \rightarrow C_{0}^{\infty}(G)$ is an isomorphism of $C_{0}^{\infty}(G)$ onto itself, see [7]. Let $\Gamma$ denote the inverse operator to $\Delta$; then $\Gamma$ is an integral operator whose kernel, $\gamma$, is the Green's kernel of $G$. This is characterised by

LEMMA 8.1. The Green's function $\gamma$ considered as a distribution on $G$ is the unique solution of the equation $\Delta \gamma=\delta-1$.

PRoof. This is taken from [7].

Let $\gamma_{\rho}$ be the distribution on $T$ given by the Fourier series

$$
\gamma_{\rho}=\sum_{B(\lambda)^{2} \neq B(\rho)^{2}} \frac{1}{B(\lambda)^{2}-B(\rho)^{2}} e^{2 \pi i \lambda},
$$

and let $J$ be the linear form on $C^{\infty}(T)$ given by

$$
J(F)=(-1)^{\mu}\left(\sum\left((-1)^{\omega} F^{\omega}\right) / j\right)^{(1)} .
$$

Here $F^{\omega}$ denotes the action of the Weyl group element $\omega$ on $F$. Since $j(x)$ divides all functions which are skew invariant under the Weyl group this is well defined. The Green's function is now given by

LEMMA 8.2. The restriction of the Green's function to $T$ is

$$
\gamma \mid T=\left(\gamma_{\rho} * J\right) / j
$$

Proof. This is again taken from [7].

By using Theorem 1.1 we can obtain another expression for $\gamma \mid T$. For the remainder of this section we shall take the heat equation in the form 
$\Delta u+\partial u / \partial t=0$ so then Theorem 1.2 gives our solution as

$$
H_{a}(x, t)=\prod_{n=1}^{\infty} \operatorname{det}\left(1-e^{-n t} \operatorname{Ad}(x)\right) \text {. }
$$

Proceeding formally we have the operator $V(t)$ defined by

$$
V(t) f(x)=e^{-t \Delta} f(x) \text {. }
$$

Since we have

$$
d(V(t) f(x)) / d t=-\Delta(V(t) f(x))
$$

then formally the kernel of $V(t)$ is convolution by $H(x, t)$. The operator $\Gamma$ can be formally obtained from $V$ by

$$
\Gamma f(x)=\int_{0}^{\infty} V(t) f(x) d t .
$$

By its definition the function $\gamma$ is the kernel of the operator $\Gamma$ and so we have obtained

Proposition 8.3. The Green's function restricted to the torus $T$ satisfies

$$
\nu * \gamma \mid T=\int_{0}^{\infty} \prod_{n=1}^{\infty} \operatorname{det}\left(1-e^{-n t} \operatorname{Ad}(x)\right) d t .
$$

Proof. Convolution with $\nu(x)$ maps $C_{0}^{\infty}(G) \rightarrow C_{0}^{\infty}(G)$ since the Haar measure on $G$ is invariant. Hence we have the composition of operators

$$
\nu * \Gamma f(x)=\left(\nu * \int_{0}^{\infty} V(t) f d t\right)(x),
$$

which gives $\nu * \gamma \mid T=\int_{0}^{\infty} H_{a}(x, t) d t$ since convolution with $H_{a}(x, t)$ is equal to the composition of applying $V(t)$ followed by convolution with $\nu(x)$.

\section{REFERENCES}

1. M. Berger, Geometry of the spectrum. I, Proc. Sympos. Pure Math., vol. 27, part 2, Amer. Math. Soc., Providence, R.I., 1975, pp. 129-152.

2. M. Berger, P. Gauduchon and E. Mazet, Le spectre d'une variété Riemannienne, Lecture Notes in Math., vol. 194, Springer-Verlag, Berlin, 1971.

3. N. Bourbaki, Groupes et algèbres de Lie, Chapitres 4, 5 et 6, Hermann, Paris, 1968.

4. F. J. Dyson, Missed opportunities, Bull. Amer. Math. Soc. 78 (1972), 635-652.

5. H. D. Fegan, The heat equation and modular forms, J. Differential Geometry (to appear).

6. H. Freudenthal and H. deVries, Linear Lie groups, Academic Press, New York, 1969.

7. S. Helgason, Fundamental solutions of invariant differential operators on symmetric spaces, Amer. J. Math. 86 (1964), 565-601.

8. in Math. 5 (1970), 1-154.

9. C. G. J. Jacobi, Fundamenta nova theoriae functionum ellipticarum, Konigsberg, 1829. Reprinted: Gesammelte Werke, Erster Band, Berlin, 1881, pp. 49-239.

10. N. Jacobson, Lie algebras, Interscience, New York, 1962.

11. B. Kostant, On Macdonald's $\eta$-function formula, the Laplacian and generalized exponents, Advances in Math. 20 (1976), 179-212. 
12. I. G. Macdonald, Affine root systems and Dedekind's $\eta$-function, Invent. Math. 15 (1972), 91-143.

13. E. T. Whittaker and G. N. Watson, $A$ course of modern analysis, Cambridge Univ. Press, Cambridge, 1920.

Department of Mathematics, Massachusetts Institute of Technology, Cambridge, MASSACHUSETTS 02139

Current address: Department of Mathematics, Rice University, Houston, Texas 77001 